\title{
COMMON EIGENVECTORS FOR COMMUTATIVE POSITIVE LINEAR OPERATORS ${ }^{1}$
}

\author{
ROBERT E. HUFF ${ }^{2}$
}

\begin{abstract}
The purpose of this note is to point out an extension of the Markov-Kakutani fixed-point theorem to a result on the existence of a common eigenvector in a cone with a compact base when acted upon by a commutative family of operators. As an application, an extension is given of a result of Kreln and Rutman on characteristic functionals.
\end{abstract}

Our terminology follows [5] and [7]. Let $K$ be a compact convex subset of a locally convex Hausdorff space, and let $A(K)$ denote the space of all real-valued, continuous, affine maps on $K$. It is known (see e.g. [1]) that the map $g: K \rightarrow\left(A(K)^{*}\right.$, weak*), where $q(k)(f)$ $=f(k)(f \in A(K), k \in K)$, is an affine homeomorphism of $K$ onto the set of all positive linear functionals on $A(K)$ of norm one. Hence, $q(K)$ is a compact base for the cone $P$ of positive linear functionals in $\left(A(K)^{*}\right.$, weak $\left.{ }^{*}\right)$. Every continuous affine map $\sigma$ on $K$ to $K$ gives rise to a unique continuous, order-preserving, linear operator $\sigma^{\prime}$ on $A(K)^{*}$ such that $\sigma^{\prime}(q(K)) \subset q(K)$ and $\sigma=q^{-1} \sigma^{\prime} q$. If $\Sigma$ is a commutative semigroup of continuous affine maps on $K$ to $K$, then the Markov-Kakutani Theorem (cf. $[4$, p. 456]) asserts the existence of a common fixed-point in $K$; equivalently, the existence of a common eigenvector, corresponding to the common eigenvalue 1 , in $P$ for the semigroup $\Sigma^{\prime}=\left\{\sigma^{\prime}: \sigma \in \Sigma\right\}$. We shall prove the following result which, by the above remarks, includes the Markov-Kakutani Theorem.

THEOREM 1. Let $E$ be an ordered, locally convex Hausdorff space whose positive cone $P$ has a compact base B. Let $\Sigma$ be a commutative semigroup of continuous, order-preserving linear operators on $E$. Then there exists a point $b_{0}$ in $B$ such that for every $\sigma$ in $\Sigma$

$$
\sigma\left(b_{0}\right)=\lambda_{\sigma} b_{0}
$$

Received by the editors July 8, 1969.

A MS Subject Classifications. Primary 4620; Secondary 4610, 4660, 1530.

Key Words and Phrases. Common eigenvectors, fixed-points, locally convex spaces, ordered linear spaces, Markov-Kakutani Theorem, compact base, characteristic functional.

1 This research is part of the author's doctoral dissertation prepared at the University of North Carolina under the direction of Professor B. J. Pettis, whose guidance is gratefully acknowledged.

2 This research was done while the author was supported by a National Science Foundation Fellowship. 
for some $\lambda_{\sigma} \geqq 0 ;$ i.e., $b_{0}$ is a common eigenvector for the members of $\Sigma$.

Note that since $B$ is a base for $P, b_{0}$ in the theorem is necessarily nonzero [7, p. 25].

The Schauder-Tychonoff fixed-point theorem will be used at an essential point in the proof.

Day [3] extended the Markov-Kakutani Theorem by replacing "commutative semigroup" by "amenable semigroup" in the hypotheses. That this is not possible in Theorem 1 is demonstrated by the following example (from $\left[8\right.$, p. 472]): consider the plane $E^{2}$ with cone $P=\{(x, y): x \geqq 0, y \geqq 0\}$ which has compact base $B=\{(x, y) \in P$ : $x+y=1\}$. Let $\Sigma$ be the multiplicative group generated by all diagonal $2 \times 2$ matrices with positive diagonal entries, together with the matrix

$$
\left[\begin{array}{ll}
0 & 1 \\
1 & 0
\end{array}\right]
$$

$\Sigma$ is a solvable group, and hence amenable (cf. [2]), but there is no common eigenvector in $B$ under the action of $\Sigma$.

Proof of Theorem 1 . Since $B$ is a base for $P$, for every element $y$ in $P$ there is a unique real number $\alpha(y) \geqq 0$ such that $y=\alpha(y) \beta(y)$ for some $\beta(y)$ in $B$; if $y$ is nonzero, then $\beta(y)$ is unique. We first show that $\alpha: y \rightarrow \alpha(y) \quad(y \in P)$ and $\beta: y \rightarrow \beta(y) \quad(y \in P, y \neq 0)$ define continuous functions on the respective domains.

(1) Suppose $k$ is a positive real number, suppose $\left\{y_{\lambda}\right\}_{\lambda}$ is a net in $[0, k] B=\{\delta b: \delta \in[0, k], b \in B\}$, and suppose $\left\{y_{\lambda}\right\}_{\lambda}$ converges to some $y$ in $P$. By compactness of $[0, k]$ and $B$, and by the Hausdorff property of $E$, every subnet of $\left\{\alpha\left(y_{\lambda}\right)\right\}_{\lambda}$ has a sub-subnet which converges to $\alpha(y)$. Hence, $\left\{\alpha\left(y_{\lambda}\right)\right\}_{\lambda}$ converges to $\alpha(y)$; and so $\alpha$ is continuous on $[0, k] B$.

Now let $y$ be any point in $P$. There is some real number $k>1$ such that $y$ is in $[0, k-1] B$. By the separation theorem (cf. $[5$, p. 119]) applied to the disjoint compact convex sets $[0, k-1] B$ and $k B$, there is a continuous linear functional $f$ on $E$ and a real number $\gamma$ such that

$$
\sup f([0, k-1] B)<\gamma<\inf f(k B) .
$$

Then $U=\{x \in P: f(x)<\gamma\}$ is a neighborhood of $y$ in $P$ and is contained in $[0, k] B$. Since $\alpha$ is continuous on $[0, k] B$, it is continuous on $U$ and hence at $y$.

(2) Suppose $\left\{y_{\lambda}\right\}_{\lambda}$ is a net in $P$ with $y_{\lambda} \neq 0$ (all $\lambda$ ), and suppose $\left\{y_{\lambda}\right\}_{\lambda}$ converges to $y \neq 0$ in $P$. By (1), $\left\{\alpha\left(y_{\lambda}\right)\right\}_{\lambda}$ converges to $\alpha(y)$. By compactness of $B$ and the fact that $\alpha(y)$ is positive, every subnet of 
$\left\{\beta\left(y_{\lambda}\right)\right\}_{\lambda}$ has a sub-subnet which converges to $\beta(y)$. Hence, $\left\{\beta\left(y_{\lambda}\right)\right\}_{\lambda}$ converges to $\beta(y)$; and so $\beta$ is continuous.

Now let $\mathcal{K}$ denote the collection of all nonvoid, compact, convex subsets $K$ of $B$ satisfying

$$
\sigma(K) \subset[0, \infty) K, \text { all } \sigma \text { in } \Sigma .
$$

If $\nVdash$ is ordered by inclusion, compactness and Zorn's Lemma imply there is some minimal member $K_{0}$ of $\varkappa$.

Choose any $\sigma$ in $\Sigma$. We argue that there is some real number $\lambda_{\sigma} \geqq 0$ such that the set

$$
K\left(\lambda_{\sigma}\right)=\left\{k \in K_{0}: \sigma(k)=\lambda_{\sigma} k\right\}
$$

is nonvoid. If $0 \in \sigma\left(K_{0}\right)$, choose $\lambda_{\sigma}=0$. If $0 € \sigma\left(K_{0}\right)$, then $\beta \circ \sigma \mid K_{0}$ is a continuous map on $K_{0}$ to $K_{0}$. By the Schauder-Tychonoff Theorem (cf. [4, p. 456]), there exists some $k_{0}$ in $K$ such that $\beta \circ \sigma\left(k_{0}\right)=k_{0}$. Then $\sigma\left(k_{0}\right)=\alpha\left(\sigma\left(k_{0}\right)\right) k_{0}$, so that we may choose $\lambda=\alpha\left(\sigma\left(x_{0}\right)\right)$.

Clearly $K\left(\lambda_{\sigma}\right)$ is compact and convex. Suppose $k$ is in $K\left(\lambda_{\sigma}\right)$. Choose any $\tau$ in $\Sigma$. By commutativity

$$
\sigma(\tau(k))=\tau(\sigma(k))=\tau\left(\lambda_{\sigma} k\right)=\lambda_{\sigma}(\tau(k)) .
$$

This implies that $\tau(k)$ is in $[0, \infty) K\left(\lambda_{\sigma}\right)$. Since $k$ was arbitrary in $K\left(\lambda_{\sigma}\right), \tau\left(K\left(\lambda_{\sigma}\right)\right) \subset[0, \infty) K\left(\lambda_{\sigma}\right)$. Since $\tau$ was arbitrary in $\Sigma, K\left(\lambda_{\sigma}\right)$ is in . By minimality of $K_{0}, K\left(\lambda_{\sigma}\right)=K_{0}$. Since $\sigma$ was arbitrary in $\Sigma$, one concludes that for every $\sigma$ in $\Sigma$ there is some $\lambda_{\sigma} \geqq 0$ such that every element $k$ in $K_{0}$ satisfies $\sigma(k)=\lambda_{\sigma} k$. Q.E.D.

As an application of Theorem 1, we extend the following result:

Theorem (KReĨN-Rutman [6, 3.3]). Suppose $E$ is an ordered, normed linear space whose positive cone $P$ is closed and has nonvoid topological interior $P^{i}$. Let $\Sigma$ be a commutative semigroup of linear operators on $E$ such that $\sigma\left(P^{i}\right) \subset P^{i}$ for each $\sigma$ in $\Sigma$. Then there exists a nontrivial, continuous, positive linear functional $f_{0}$ on $E$ such that for every $\sigma$ in $\Sigma$

$$
f_{0} \circ \sigma=\lambda_{\sigma} f_{0}
$$

for some scalar $\lambda_{\sigma}>0$.

If a linear space $E$ is ordered by a cone $P$, then $P^{0}$ will denote the set of all order units in $P[7, \mathrm{p} .4] . P^{0}$ is the "radial kernel" of $P$ in the terminology of $[5$, p. 14] (the set of all "internal points" of $P$ in the terminology of $[4, \mathrm{p} .410]) . P^{0}$ equals the topological interior $P^{i}$ of $P$ whenever $E$ is a linear topological space and $P^{i}$ is nonvoid (cf. $[4, \mathrm{p}$. 
$413]$ ). If $P^{0}$ is nonvoid, every nonzero positive linear functional on $E$ is strictly positive at every member of $P^{0}$.

Theorem 2. Suppose $E$ is an ordered linear space such that the positive cone $P$ has nonvoid radial kernel $P^{0}$. Let $\Sigma$ be a commutative semigroup of order-preserving, linear operators on $E$. Then there exists a nontrivial positive linear functional $f_{0}$ on $E$ such that for every $\sigma$ in $\Sigma$

$$
f_{0} \circ \sigma=\lambda_{\sigma} f_{0}
$$

for some $\lambda_{\sigma} \geqq 0$.

If $\sigma$ is in $\Sigma$ and if $\sigma\left(P^{0}\right) \cap P^{0}$ is nonvoid, then $\lambda_{\sigma}>0$.

If $\sigma$ is in $\Sigma$ and if $\sigma\left(y_{0}\right)=\mu_{\sigma}\left(y_{0}\right)$ for some $y_{0}$ in $P^{0}$ and some scalar $\mu_{\sigma}$, then $\lambda_{\sigma}=\mu_{\sigma}$. In particular, if for every $\sigma$ in $\Sigma$ there is some $\sigma$-fixed-point in $P^{0}$, then $f_{0}$ is a common fixed-point for the adjoints of the members of $\Sigma$.

As an example of a situation satisfying the hypotheses of Theorem 2, but not those in the Krein-Rutman Theorem, take the nonnegative, diagonal $2 \times 2$ matrices acting on the plane ordered by the cone $\{(x, y): x \geqq 0, y \geqq 0\}$.

Proof of Theorem 2. Let $E^{\prime}$ denote the algebraic dual of $E$, let $E^{\prime}$ have the topology of pointwise convergence on $E$, and let $P^{\prime}$ denote the cone of all positive linear functionals on E. $P^{\prime}$ is nontrivial (cf. $\left[5\right.$, p. 23, (3.2)]). The adjoint $\sigma^{\prime}$ of a member $\sigma$ of $\Sigma$ is continuous, linear, and maps $P^{\prime}$ into $P^{\prime}$. Let $p_{0}$ be any point in the radial kernel of $P$, and let $B^{\prime}=\left\{f \in P^{\prime}: f\left(p_{0}\right)=1\right\}$. Then $B^{\prime}$ is a base $P^{\prime}$. Furthermore, since for every $y$ in $E$ there exists a real number $\delta_{y}>0$ such that

$$
-\delta_{y} p_{0} \leqq y \leqq \delta_{y} p_{0}
$$

in $E, B^{\prime}$ is homeomorphic to a closed subset of the product space $\prod\left\{\left[-\delta_{y}, \delta_{y}\right]: y \in E\right\}$. Thus, $B^{\prime}$ is a compact base for $P^{\prime}$. By Theorem 1 , there exists an element $f_{0}$ in $B^{\prime}$ such that for every $\sigma$ in $\Sigma$

$$
f_{0} \circ \sigma=\sigma^{\prime}\left(f_{0}\right)=\lambda_{\sigma} f_{0}
$$

for some $\lambda_{\sigma} \geqq 0$.

Suppose $\sigma$ is in $\Sigma$ and $y_{0}$ is in $\sigma\left(P^{0}\right) \cap P^{0}$. Then $f_{0} \circ \sigma\left(y_{0}\right)>0$ and $f_{0}\left(y_{0}\right)>0$, so that $\lambda_{\sigma}>0$.

Suppose $\sigma$ is in $\Sigma$ and the $y_{0}$ is some point in $P^{0}$ such that $\sigma\left(y_{0}\right)$ $=\mu_{\sigma} y_{0}$ for some scalar $\mu_{\sigma}$. Then

$$
\lambda_{\sigma} f_{0}\left(y_{0}\right)=f_{0} \circ \sigma\left(y_{0}\right)=f_{0}\left(\mu_{\sigma} y_{0}\right)=\mu_{\sigma} f_{0}\left(y_{0}\right) .
$$

Since $f_{0}\left(y_{0}\right)>0, \lambda_{\sigma}=\mu_{\sigma}$. Q.E.D.

Finally, we remark that, following Silverman and Yen's modifica- 
tion of the Kreinn-Rutman result [8], one can weaken slightly the assumption of commutativity in both Theorem 1 and Theorem 2. If one replaces commutativity of $\Sigma$ in Theorem 1 by the following assumption:

(A1) there is a subset $S$ of $\Sigma$ such that

(1) there is some point $\bar{b}$ in $B$ satisfying $s(\bar{b})=\bar{b}$ for every $s$ in $S$, and

(2) for every pair $\sigma_{1}, \sigma_{2}$, in $\Sigma$ there are elements $s_{1}, s_{2}$ in $S$ such that $\sigma_{1} \sigma_{2} s_{1}=\sigma_{2} \sigma_{1} s_{2}$,

then the set $B_{1}=\{b \in B: s(b)=b$ for every $s$ in $S\}$ is a compact base for the cone $P_{1}=[0, \infty) B_{1}$ which it generates. Using (A1)(2), it can be shown that $\sigma\left(P_{1}\right) \subset P_{1}$ for every $\sigma$ in $\Sigma$, and that the elements of $\Sigma$ commute on the linear span of $P_{1}$. Hence, the conclusion of Theorem 1 remains valid if the assumption of commutativity of $\Sigma$ is replaced by assumption (A1). Using this extended result in the proof of Theorem 2 , we have that the conclusions of Theorem 2 remain valid if the assumption of commutativity of $\Sigma$ is replaced by the following assumption:

(A2) there is a subset $S$ of $\Sigma$ such that

(1) there is some nonzero $f_{1}$ in $P^{\prime}$ satisfying $f_{1} \circ s=f_{1}$ for all $s$ in $S$, and

(2) for every pair $\sigma_{1}, \sigma_{2}$ in $\Sigma$ there are elements $s_{1}, s_{2}$ in $S$ such that $s_{1} \sigma_{1} \sigma_{2}=s_{2} \sigma_{2} \sigma_{1}$.

\section{REFERENCES}

1. L. N. Argabright, Invariant means and fixed points: $A$ sequel to Mitchell's paper, Trans. Amer. Math. Soc. 130 (1968), 127-130. MR 36 \#667.

2. M. M. Day, Amenable semigroups, Illinois J. Math. 1 (1957), 509-544. MR $19,1067$.

3. - Fixed point theorems for compact convex sets, Illinois J. Math. 5 (1961), 585-590. MR $25 \# 1547$.

4. N. Dunford and J. T. Schwartz, Linear operators. I: General theory, Pure and Appl. Math., vol. 7, Interscience, New York, 1958. MR 22 \#8302.

5. J. L. Kelley, I. Namioka, et al, Linear topological spaces, The University Series in Higher Math., Van Nostrand, Princeton, N. J., 1963. MR 29 \#3851.

6. M. G. Kreln and M. R. Rutman, Linear operators leaving invariant a cone in a Banach space, Uspehi Mat. Nauk 3 (1948), no. 1 (23), 3-95; English transl., Amer. Math. Soc. Transl. (1) 10 (1962), 199-325. MR 10, 256.

7. A. L. Peressini, Ordered topological vector spaces, Harper \& Row, New York, 1967. MR 37 \#3315.

8. R. J. Silverman and T. Yen, Characteristic functionals, Proc. Amer. Math. Soc. 10 (1959), 471-477. MR 22 \#187.

University of North Carolina, Chapel Hill, North Carolina 27514 